\title{
Algorithm design for a supply chain equilibrium management model
}

\author{
Peimin Zhao \\ Feixian School \\ Linyi University \\ Feixian, Shandong, P.R.China
}

\begin{abstract}
In this paper, we consider a complementary model for the equilibrium management of supply chain. In order to give an optimal decision for the equilibrium management, we propose a new algorithm based on an estimate of the error bound. This algorithm requires neither the existence of a non-degenerate solution nor the non-singularity of the Jacobian matrix at the solution. We also prove the quadratic convergence of the given algorithm. It can be viewed as extensions of previously known results.
\end{abstract}

Keywords- Supply chain equilibrium management; complementary model; algorithm; quadratic convergence.

\section{INTRODUCTION}

Supply chain management involves many aspects, including manufacturing, transportation, logistics and sales markets, etc. Manufacturers produce a commodity and then transport it to sellers. The manufacturers need to pay for the costs of production and transportation. In order to make a profit, they have to determine optimal production and transportation. Sellers need to pay for the costs in stores, and maximize their profits from the commodity price. Commodity price is the price customers are willing to pay. The customers need to determine their optimal level of consumption, which is related to commodity prices at different stages of the supply chain and the commodity handling costs. The supply chain management problem can be abstracted as a "complementary" relationship between the two sets of decision variables, expressed as a complementary model ([1,2,3]).

Let mapping $f: R^{n} \rightarrow R^{n}$, be continuously differentiable. Find vector $x^{*} \in R^{n}$, such that

$$
x \geq 0, f(x) \geq 0, x^{T} f(x)=0,
$$

We use $X^{*}$ to denote the solutions of (1), and assume that $X^{*}$ is not empty.

The model takes into consideration different behaviors and their interactions of the various participants, and provides a standard to assess the appropriate commodity prices and quantities. It is an equilibrium optimization problem. At its core is the use of mathematical methods, with computer and network as tools, to study various complex systems having this complementary relationship and their corresponding solutions. It provides scientific basis for decision makers, with the goal of ultimately achieving balanced and harmonious development of complex system operation. It is widely used nowadays. In recent years it has become a hot topic of operational research and management studies. Many researchers, especially scholars abroad, are increasingly interested, and great progress has been made in both theoretical research and practical applications. To solve (1), many algorithms have been proposed ([4, 5, 6, 7, 9]). The basic idea of these algorithms is to transform (1) into an unconstrained optimization problem, or into a simple constrained optimization problem $([4,7,9])$, and then to use the Newton type algorithms or confidence region algorithms. Although the convergence and the corresponding rate of convergence are established, the conditions are mostly quite strong. Most of these algorithms require that the Jacobian matrix at the solution of (1) is non-singular or there exists a non-degenerate solution. As we know, the non-singularity of the Jacobian matrix at the solution of (1) ensures that the application of the well-known Levenberg-Marquardt (L-M) algorithm for solving (1) has the property of quadratic convergence ([7]). Recently, Yamashita, and Fukushima have proved that under certain conditions on the local error bound, the L-M algorithm for solving nonlinear equations also has the property of quadratic convergence. These conditions on local error bound are weaker than the non-singularity of the Jacobian matrix ([8]). This motivates us to transform (1) into a nonlinear equation, and to establish the error bound for (1). With the establishment of an error bound of a residual function from (1), we propose a new algorithm and prove its quadratic convergence, thereby overcoming the drawbacks of the above mentioned algorithms on convergence.

\section{PRELIMINARIES}

In this section, we mainly give a result on the error bound for (1) ([10]), which play a key role in the establishment of the nonlinear equation and in the proof of the convergence of the proposed algorithm.

Assumption 1 Let $f: R^{n} \rightarrow R^{n}$ be a uniform $P$ function, i.e., there exists a constant $\mu>0$ such that

$$
\max _{1 £ i £ n}\left\{(x-y)_{i}[f(x)-f(y)]_{i}\right\}^{3} m\|x-y\|^{2}, " x, y \hat{\mathrm{I}} R^{n} \text {. }
$$

Theorem 1 Assume that the mapping $f: R^{n} \rightarrow R^{n}$ in (1) is a uniform $P$ function, and is Lipschitz continuous. Then there exist constant $\eta_{1}>0$, and $x^{*} \in X^{*}$ for any $x \in R^{n}$, such that $\left\|x-x^{*}\right\| \leq \eta_{1}\|\min \{x, f(x)\}\|$. 


\section{ALGORITHMS AND CONVERGENCE}

In this section, we first transform (1) into a nonlinear equation, and then give another type of error bound for (1). Based on this, we give the new algorithm for solving (1) and establish its property of quadratic convergence, overcoming the drawbacks of requiring the non-singularity of the Jacobian matrix at the solution or the existence of a non-degenerate solution.

We transform (1) into a nonlinear equation via Fischer function ([11]) $\phi: R^{2} \rightarrow R^{1} \phi(a, b)=\sqrt{a^{2}+b^{2}}-a-b, a, b \in R$.

This function has the property $\phi(a, b)=0 \Leftrightarrow a \geq 0, b \geq 0$, $a b=0$. For any $(a, b) \in R^{2}$, Fischer function also has the following property ([12])

$$
(2-\sqrt{2})|\min \{a, b\}| \leq|\phi(a, b)| \leq(2+\sqrt{2})|\min \{a, b\}| .
$$

For any vectors $a, b \in R^{n}$, we define the following vectorvalued function $\Phi(a, b)=\left(\phi\left(a_{1}, b_{1}\right), \phi\left(a_{2}, b_{2}\right), \cdots, \phi\left(a_{n}, b_{n}\right)\right)^{T}$,

Where $a=\left(a_{1}, a_{2}, \cdots, a_{n}\right)^{T}, b=\left(b_{1}, b_{2}, \cdots, b_{n}\right)^{T}$. Obviously, $\square$

$$
\Phi(a, b)=0 \Leftrightarrow a \geq 0, b \geq 0, a^{T} b=0 .
$$

With (2), (3) and Theorem 1, we can easily prove the following result.

Theorem 2 If the mapping $f: R^{n} \rightarrow R^{n}$ in (1) is a uniform $P$ function, and is Lipschitz continuous, then there exist constant $\eta_{2}>0$, and $x^{*} \in X^{*}$ for any $x \in R^{n}$, such that $\left\|x-x^{*}\right\| \leq \eta_{2} \Phi(x, f(x))$.

Function (4) is not smooth. In order to give a smooth algorithm with the property of quadratic convergence, we give also the following smooth Fisher-Burmeister function $\varphi_{\tau}: R^{2} \rightarrow R^{1} \varphi_{\tau}(a, b)=\sqrt{a^{2}+b^{2}+2 \tau^{2}}-a-b$, where $\tau>0$ is a smooth parameter. For ease of presentation, let

$$
\Theta_{\tau}(y, z)=\left(\varphi_{\tau}\left(y_{1}, z_{1}\right), \varphi_{\tau}\left(y_{2}, z_{2}\right), \cdots, \varphi_{\tau}\left(y_{n}, z_{n}\right)\right)^{T} \in R^{n},
$$

We define the following vector-valued function

$$
F: R^{n} \times(0, \infty) \rightarrow R^{n} \times(0, \infty), \quad F(x, \tau):=\left(\begin{array}{c}
\Theta_{\tau}(x, f(x)) \\
\tau
\end{array}\right)
$$

and real-valued function $f: R^{n+1} \rightarrow R$

$$
f(x, \tau):=F(x, \tau)^{T} F(x, \tau)=\|F(x, \tau)\|^{2} .(6)
$$

Obviously, $x^{*} \in X^{*} \Leftrightarrow\left(x^{*}, 0\right) \square$ is a solution of $F(x, \tau)=0$ .In the following, we give the algorithm for solving

$F(x, \tau)=0$, and denote the solutions of $F(x, \tau)=0$ by $\Omega^{*}$.

First, we give some properties of function $\varphi_{\tau}(a, b)$ ([13], [14])

Lemma 1 Function $\varphi_{\tau}(a, b)$ has the following properties: (i) Function $\varphi_{\tau}(a, b)$ is continuously differentiable on $R^{2} \times(0, \infty)$; function $\varphi_{\tau}(a, b)$ is strongly semi-smooth on $(a, b, \tau) \in R^{2} \times[0, \infty)$ that is,

$$
\varphi_{(\tau+\Delta \tau)}(a+\Delta a, b+\Delta b)-\varphi_{\tau}(a, b)-V^{T}(\Delta a, \Delta b, \Delta \tau)=O\left(\|(\Delta a, \Delta b, \Delta \tau)\|^{2}\right)
$$

Where $V \in \partial \varphi_{(\tau+\Delta \tau)}(a+\Delta a, b+\Delta b)$, and $(\Delta a, \Delta b, \Delta \tau) \rightarrow 0$, $\partial \varphi$ is the Clarke generalized gradient of $\varphi$ ([15]) .

(ii) For any $(a, b, \tau) \in R^{2} \times(0, \infty)$, we have

$$
\left|\varphi_{0}(a, b)-\varphi_{\tau}(a, b)\right| \leq \sqrt{2} \tau .
$$

For (5), using Lemma 1, we have the following result.

Theorem 3 Function $F(x, \tau)=0$ has the following properties:

(i)Function $F(x, \tau)$ is continuously differentiable on $R^{n} \times(0, \infty) \square \square$ and is locally Lipschitz continuous and strongly semi-smooth, i.e., for any $(x, \tau) \in R^{n} \times[0, \infty)$, there exist $L_{1}>0, L_{2}>0$ and $b_{1}>0$ such that

$$
\begin{gathered}
\|F(x+\Delta x, \tau+\Delta \tau)-F(x, \tau)\| \leq L_{1}\|(\Delta x, \Delta \tau)\| \\
\left\|F(x+\Delta x, \tau+\Delta \tau)-F(x, \tau)-V^{T}(\Delta x, \Delta \tau)\right\| \leq L_{2}\|(\Delta x, \Delta \tau)\|^{2},
\end{gathered}
$$

$\forall(\Delta x, \Delta \tau) \in N\left(0, b_{1}\right):=\left\{(\Delta x, \Delta \tau) \in R^{n} \times[0, \infty)\|\|(\Delta x, \Delta \tau) \| \leq b_{1}, \tau+\Delta \tau \geq 0\right\}$,

Where $V \in \partial F(x+\Delta x, \tau+\Delta \tau), \quad \partial F(x, \tau)$ is the Clarke generalized gradient of $F(x, \tau)$.

(ii) From Theorem 2, for a solution $\left(x^{*}, 0\right) \in \Omega^{*}$, there exist a neighbourhood $N\left(\left(x^{*}, 0\right), b_{2}\right) \in \Omega^{*}$ of $\left(x^{*}, 0\right)$ and a constant $c_{1}>0$, such that for any $(x, \tau) \in N\left(\left(x^{*}, 0\right), b_{2}\right)$, we have

$$
\operatorname{dist}\left((x, \tau), \Omega^{*}\right) \leq c_{1}\|F(x, \tau)\|,(9)
$$

Where $N\left(\left(x^{*}, 0\right), b_{2}\right):=\left\{(x, \tau)\left\|\mid(x, \tau)-\left(x^{*}, 0\right)\right\| \leq b_{2}, \tau \geq 0\right\}$.

Proof . (i) is a direct result of Lemma 1.

(ii) From Theorem 2, there exists a constant $1>b_{2}>0$, such that $\operatorname{dist}\left(x, X^{*}\right) \leq \eta_{3}\|\Psi(x)\|, \forall x \in N\left(x^{*}, b_{2}\right)$, where $N\left(x^{*}, b_{2}\right):=\left\{x \in R^{n}\left\|x-x^{*}\right\| \leq b_{2}\right\}$, and there exists $\bar{x} \in X^{*}$, such that $\operatorname{dist}\left(x, X^{*}\right)=\|x-\bar{x}\|$, From Lemma 1(ii), we have

$$
\begin{aligned}
& \left\|\Theta_{0}(x, f(x))\right\|-\left\|\Theta_{\tau}(x, f(x))\right\| \leq\left\|\Theta_{\tau}(x, f(x))-\Theta_{0}(x, f(x))\right\| \\
= & \left(\sum_{i=1}^{s}\left(\varphi_{\tau}\left(x_{i}, f(x)_{i}\right)-\varphi_{0}\left(x_{i}, f(x)_{i}\right)^{2}\right)^{\frac{1}{2}} \leq \sqrt{2 n} \tau .\right.
\end{aligned}
$$

Because of this, for any $(x, \tau) \in N\left(\left(x^{*}, 0\right), b_{2}\right)$ we have $\operatorname{dist}\left(x, X^{*}\right)=\|x-\bar{x}\| \leq\|(x, \tau)-(\bar{x}, 0)\| \leq\|x-\bar{x}\|+\tau \leq \eta_{3}\|\Phi(x)\|+\tau$ 


$$
\begin{aligned}
& =\eta_{3}\left\|\Theta_{0}(x, f(x))\right\|+\tau \leq \eta_{3}\left(\left\|\Theta_{\tau}(x, f(x))\right\|+\left(\sqrt{2 n} \eta_{3}+1\right) \tau\right. \\
& \leq\left(\sqrt{2 n} \eta_{3}+1\right)\left(\left\|\Theta_{\tau}(x, f(x))\right\|+\tau\right) \\
& \leq\left(\sqrt{2 n} \eta_{3}+1\right)\left(\left\|\Theta_{\tau}(x, f(x))\right\|_{1}+\tau\right) \leq\left(\sqrt{2} n \eta_{3}+1\right)\|F(x, \tau)\|_{1} \\
\leq & \sqrt{n+1}\left(\sqrt{2} n \eta_{3}+1\right)\|F(x, \tau)\| .
\end{aligned}
$$

In the following we give a smooth Levenberg-Marquardt algorithm for solving (1).

\section{Algorithm:}

Step 1: Select an initial point $x^{0} \in R^{n}$, and a parameter

$\tau^{0}>0 \quad \varepsilon>0 . \quad$ Let $k=0$.

Step 2: If $\left\|\nabla f\left(x^{k}, \tau^{k}\right)\right\| \leq \varepsilon$, stop; otherwise, go to Step 3 .

Step 3: Choose $H^{k}$, where $H^{k}$ is the Jacobian matrix of $F\left(x^{k}, \tau^{k}\right), \mu^{k}=\left\|F\left(x^{k}, \tau^{k}\right)\right\|^{2}$. Let $d^{k}=\left(\Delta x^{k}, \Delta \tau^{k}\right) \in R^{n+1}$ be the solution of the following strictly convex quadratic programming

$$
\begin{array}{lc}
\min & \left\|F\left(x^{k}, \tau^{k}\right)+H^{k} d\right\|^{2}+\mu^{k}\|d\|^{2} \\
\text { s.t. } & |\nabla \tau| \leq \frac{1}{1+\mu^{k}} \tau^{k}
\end{array}
$$

Step 4: Let $x^{k+1}:=x^{k}+\Delta x^{k}, \tau^{k+1}:=\tau^{k}+\Delta \tau^{k}, k:=k+1$, go to Step 2 .

In the following convergence analysis, we assume that Algorithm 1 generates an infinite sequence. From (7)-(9), combined with (14) - (15), and the proof of Theorem 2.1 ([8]), we can obtain the quadratic convergence rate of Algorithm 1.

Theorem 4 Let $\left\{\left(x^{k}, \tau^{k}\right)\right\}$ be an infinite sequence generated by Algorithm 1. If $\left(x^{0}, \tau^{0}\right)$ is close to $\left(x^{*}, 0\right)$ sufficiently, then $\operatorname{dist}\left(\left(x^{k}, \tau^{k}\right), \Omega^{*}\right)$ converges to 0 quadratically, and sequence $\left(x^{k}, \tau^{k}\right)$ converges quadratically to $\left(\$_{0}, 0\right) \in \Omega^{*} \mathrm{I} N\left(\left(x^{*}, 0\right), b_{2} / 2\right)$.

\section{CONCLUSION AND PROSPECT}

In this paper, we present a new algorithm for solving the equilibrium management model of supply chain, and also have showed that method has the quadratic convergence, the conditions guaranteeing the quadratic convergence in this paper are weaker than those in the existing resolving methods in [7], since it does not require the existence of a nondegenerate solution of (1), nor the non-singularity of the Jacobian matrix at the solution of (1),
It is uncertain whether we have that the results in this paper for the functions which contain the $P$ functions discussed in this paper, this is a topic for further research.

\section{ACKNOWLEDGMENT}

The authors wish to give their sincere thanks to the editor and the anonymous referees for their valuable suggestions and helpful comments which improved the presentation of the paper.

This work was supported by Shandong Provincial Natural Science Foundation (ZR2010AL005, ZR2011FL017), and the projects for reformation of Chinese universities logistics teaching and research (JZW2012065).

\section{REFERENCES}

[1] M.C.Ferris and J.S. Pang. Engineering and economic applications of complementarity problems. Society for industrial and applied mathematics, 1997, 39(4), 669-713.

[2] A. Nagurney, J. Dong, D. Zhang. A supply chain network equilibrium model. Transportation Research, 2002, Part E 38, 281.

[3] L.P. Zhang. A nonlinear complementarity model for supply chain network equilibrium. Journal of Industrial and Managment Optimization, 2007, 3(4), 727-737.

[4] R. Andreani, A. Friedlander and S.A. Santos. On the resolution of the generalized nonlinear complementarity problem. SIAM Journal on Optim., 2001, 12, 303-321.

[5] F. Facchinei and J.S. Pang. Finite-Dimensional Variational Inequality and Complementarity Problems. Springer, New York, 2003.

[6] C. Kanzow and M. Fukushima. Equivalence of the generalized complementarity problem to differentiable unconstrained minimization. J. Optim. Theory and Appl., 1996, 90(3),581-603.

[7] Y.J. Wang, F.M. Ma and J.Z. Zhang. A nonsmooth L-M method for solving the generalized nonlinear complementarity problem over a polyhedral cone. Appl. Math. Optim., 2005, 52(1), 73-92.

[8] N. Yamashita and M. Fukushima. On the rate of convergence of the Levenberg-Marquardt method. Computing [Suppl]\}, 2001, 15, 239-249.

[9] X.Z. Zhang, F.M. Ma and Y.J. Wang. A Newton-type algorithm for generalized linear complementarity problem over a polyhedral cone. Appl. Math. Comput., 2005, vol.169, 388-401.

[10] N.H. Xiu and J.Z. Zhang. Global projection-type error bound for general variational inequlities. J. Optim. Theory Appl., 2002, 112(1), 213-228.

[11] A. Fischer. A special Newton-type optimization method. Optim., 1992, $24,269-284$.

[12] P. Tseng. Growth behavior of a class of merit function for the nonlinear complementarity problem. J. Optim.Theory Appl., 1996, 89, 17-37.

[13] S. Engelke, C. Konzow. Improved smoothing type method for the solution of linear programs. Preprint, Institute of Applied Mathematics, University of Hamburg, Hamburg, March, 2000.

[14] L. Qi, D. Sun. Smoothing function and smoothing Newton method for complmentarity problems and variational inequality problems. Reprot, University of New South Wales, Sydney, Australia, 1998.

[15] F.H. Clarke. Optimization and Nonsmooth Analysis. John Wiley and Sons, New York, NY, 1983. 